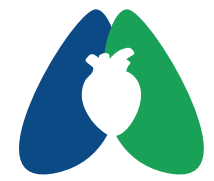

ASSOBRAFIR C I Ê N C I A

\section{Teste de se levantar e sentar de um minuto em adolescentes com fibrose cística: qual 0 desempenho na exacerbação pulmonar aguda? Uma série de casos*}

\author{
The 1-min sit-to-stand test in adolescents with cystic fibrosis: \\ What is the performance during acute pulmonary exacerbation? \\ Case series
}

Tayna Castilho ${ }^{1}$ (1); Juliana Cardoso ${ }^{1}$ (D); Renata Maba Gonçalves Wamosy² (1); Camila Isabel Santos Schivinski ${ }^{10}$

\begin{abstract}
Resumo
Introdução: A capacidade de exercício de indivíduos com fibrose cística (FC) é um importante marcador clínico, que apresenta correlação com qualidade de vida e mortalidade nessa população. Sendo assim, se torna relevante a investigação desse marcador clínico durante exacerbação pulmonar aguda (EPA). Objetivo: Descrever o desempenho no teste de se levantar e sentar de um minuto (TLS), a função pulmonar e a mecânica respiratória de adolescentes com FC, no início e ao final do regime de internação para tratamento da EPA. Métodos: Série de casos que incluiu adolescentes entre 12 e 14 anos com FC que estavam hospitalizados por EPA. Os dados foram obtidos no início e final da internação (dados antropométricos, clínicos e de parâmetros do sistema respiratório); também foram aplicados escores específicos de EPA e o TLS. No TLS foram registrados parâmetros cardiorrespiratórios no início e ao final do teste. Utilizou-se estatística descritiva para apresentação dos dados. Resultados: Participaram 4 adolescentes com média de idade de 13 $\pm 0,81$ anos. Houve melhora dos escores de exacerbação, bem como dos parâmetros do sistema respiratório ao final da internação. O desempenho médio no TLS apresentou incremento de 20,2 $\pm 13,3$ repetições para $28,5 \pm 14,7$ ao final da internação, porém sem mudanças individuais nos parâmetros cardiorrespiratórios. Conclusão: O tratamento da EPA provocou melhora nos parâmetros do sistema respiratório, bem como da capacidade de exercício dos adolescentes com FC que participaram desta série de casos.
\end{abstract}

Palavras-chave: Fibrose Cística; Exacerbação Pulmonar; Função Pulmonar; Capacidade de Exercício.

\begin{abstract}
Background: the exercise capacity in individuals with cystic fibrosis (CF) is an important clinical marker, which correlates to quality of life and mortality in this population. Therefore, investigate this clinical marker during acute pulmonary exacerbation (APE) is relevant. Aim: to describe the performance on the 1-min sit-to-stand test (STS), the pulmonary function and the respiratory mechanics at the beginning and at the end of hospitalization for APE. Methods: case series that included adolescents between 12 to 14 years old with $\mathrm{CF}$, and were hospitalized with APE. At the beginning and at the end of hospitalization were obteined data of weight, height, body mass index, disease severity and respiratory system parameters (Impulse Oscillometry-IOS, Spirometry); also, were applied specific scores of APE and the STS. At the STS were registered cardiorespiratory parameters at the beginning and at the end of the test. The data were analyzed with descriptive statistics. Results: participated 4 adolescents ( $13 \pm 0.81$ years old). There was improvement on the APE scores and the respiratory parameters at the end of the hospitalization. The mean performance on STS presented improvement of $20.2 \pm 13.3$ repetitions to $28.5 \pm 14.7$ at the end of the hosptalization; however, the cardiorespiratory parameters did not show individual changes. Conclusion: the treatment of APE improved the respiratory system parameters and the exercise capacity of adolescents with CF that participated in this case series.
\end{abstract}

Keywords: Cystic Fibrosis; Pulmonary Exacebation; Pulmonary Function; Exercise Capacity.
*Autor correspondente:
Camila Isabel Santos Schivinski. E-mail:
cacaiss@yahoo.com.br do Estado de Santa Catarina (UDESC) (HIJG), Florianópolis, SC, Brasil.

83106817.0 .0000 .0118 da Universidade do Estado de Santa Catarina, n².710.712. da Saúde e do Esporte (CEFID), Universidade

Fisioterapia, Centro de Ciências da Saúde e

resumo com resultados parciais (n) 19/10/2019, na cidade de Gramado/

Submissão em: Fevereiro 23, 2021

Aceito em: Abril 05, 2021

Este é um artigo publicado em acesso aberto (Open Access) e distribuído sob a licença Creative Commons Attribution NonComercial ShareAlike License, que permite uso, distribuição e reprodução em qualquer meio, sem restrições desde que sem fins comerciais e que o trabalho original seja corretamente citado e de forma que não indique endosso ao trabalho feito. Adicionalmente, qualquer trabalho derivado deverá ser publicado sob a mesma licença. 


\section{INTRODUÇÃO}

A capacidade de exercício é considerada um marcador clínico importante na fibrose cística (FC) e, sua melhora ou manutenção, é um dos objetivos do acompanhamento fisioterapêutico durante a progressão da doença ${ }^{1,2}$. Isso porque já se sabe que existe relação entre esse desfecho, a qualidade de vida e a taxa de mortalidade na FC'1. Sendo assim, o guideline Cystic Fibrosis Trust recomenda que a avaliação da capacidade de exercício deve ser realizada antes e após mudanças ou intervenções significativas². Isso remete que esse marcador deva ser incluído como parâmetro de resposta ao regime terapêutico para tratamento da exacerbação pulmonar aguda (EPA).

Para a avaliação da capacidade de exercício, a Recomendação Brasileira de Fisioterapia na FC sugere avaliação do consumo máximo de oxigênio ou pico obtidos durante teste de exercício cardiopulmonar (TECP), o qual é considerado padrão-ouro ${ }^{1}$. Entretanto, um teste mais viável para avaliar a capacidade de exercício durante regime de internação para tratamento da EPA é o teste de se levantar e se sentar (TLS), o qual também é recomendado por guidelines de fisioterapia ${ }^{1,2}$, mas que ainda não foi reportado nessa condição de saúde na população pediátrica com FC. O TLS é um teste de campo que foi desenvolvido para indivíduos com essa doença e tem como objetivo avaliar a capacidade anaeróbica e a força muscular de membros inferiores, visto que esses desfechos estão relacionados com a capacidade aeróbica. O indivíduo deve levantar e se sentar em uma cadeira repetidamente durante um minuto para sua realização ${ }^{3}$.

Diante do que foi exposto, o objetivo deste relato de série de casos foi descrever o desempenho no TLS, a função pulmonar e a mecânica respiratória de adolescentes com FC, no início e ao final do regime de internação para tratamento da EPA.

\section{MÉTODOS}

Essa série de casos incluiu pacientes com FC entre 12 e 14 anos, internados no centro de referência de FC por 14 dias para tratamento da EPA. A pesquisa foi aprovada pelo Comitê de Ética Pesquisa da da Universidade do Estado de Santa Catarina e do referido centro de referência (CAAE: 83106817.0.0000.0118). Foi obtida assinatura do termo de consentimento livre e esclarecido dos adolescentes e responsáveis, além da assinatura do termo de assentimento. A coleta de dados ocorreu no período de Junho de 2018 até Março de 2019.

\section{COLETA DE DADOS}

Em prontuário, foram obtidos dados de colonização por patógenos, genótipo e gravidade da doença (Escore de Schwachman-Doershuk-ESD). A coleta de dados ocorreu nas primeiras e últimas 72 horas de internação, em que se aplicou os escores CFCS e CFFS para confirmar a presença de EPA, e realizou-se avaliação do sistema respiratório por meio do sistema de oscilometria de impulso - IOS e espirometria (Master Screen IOS, Erich Jaeger, Germany®). Os parâmetros respiratórios foram apresentados em porcentagem do predito (\%), segundo Assumpção et al. ${ }^{4}$ para o IOS, e de acordo com Polgar et al. ${ }^{5}$ e Knudson et al. ${ }^{6}$ para os parâmetros espirométricos. Por fim, conduziuse a aplicação do TLS durante um minuto ${ }^{3}$, em que os escolares foram orientados a levantar e se sentar em uma cadeira o maior número de vezes, controlando-se parâmetros cardiorrespiratórios, no início e ao final do teste: de frequência cardíaca (fc), frequência respiratória $(\mathrm{fr})$, saturação de pulso de oxigênio $\left(\mathrm{SpO}_{2}\right)$ e percepção de esforço por meio da escala de BORG modificada e a escala de percepção de esforço para criança (EPEC). A fc ao final do teste foi usada para calcular a fc máxima predita para a idade, validada para a população brasileira .

Os dados de cada caso foram armazenados no software IBM SPSS-20.0 $0^{\circ}$ e realizou-se análise descritiva dos dados, os quais foram apresentados em tabelas com informações relacionadas ao início e final da internação.

\section{RESULTADOS}

Participaram 4 adolescentes (50\% meninas) com média

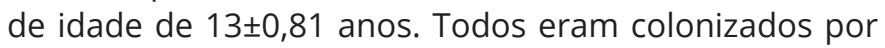
algum patógeno e com genotipagem diferente da mutação $\triangle$ F508 (tabela 1). Além disso, para o tratamento da EPA, todos os adolescentes realizaram antibioticoterapia intravenosa, suporte nutricional e intensificação da inalação de mucolíticos e fisioterapia respiratória.

Na tabela 2 são apresentados os dados dos escores de exacerbação, parâmetros da espirometria e IOS, no

Tabela 1. Principais características da amostra (dados demográficos e clínicos).

\begin{tabular}{|c|c|c|c|c|c|c|}
\hline Caso & Idade (anos) & Sexo & S. aureus & P. aerugionosa & B. серасia & $\begin{array}{l}\text { ESD pontos } \\
\text { (classificação) }\end{array}$ \\
\hline 1 & 14 & $\mathrm{~F}$ & Sim & Não & Sim & 55 pontos (moderada) \\
\hline 2 & 13 & M & Sim & Sim & Não & 65 pontos (médio) \\
\hline 3 & 13 & M & Não & Sim & Não & 95 pontos (excelente) \\
\hline 4 & 12 & $\mathrm{~F}$ & Não & Não & Sim & 60 pontos (médio) \\
\hline
\end{tabular}

Legenda: São apresentados os dados individuais de cada caso. F: feminino; M: masculino; S. aureus: Staphylococcus aureus; P. aeroguginosa: Pseudomonas aeruginosa; B. cepacia: Burkholderia cepacia; ESD: escore de Schwachman-Doershuk. 
Tabela 2. Apresentação dos dados antropométricos, escores de exacerbação e parâmetros respiratórios no início e final da internação por EPA.

\begin{tabular}{|c|c|c|c|c|c|c|c|c|c|c|c|c|}
\hline \multirow{2}{*}{ Parâmetros } & \multicolumn{3}{|c|}{ Caso 1} & \multicolumn{3}{|c|}{ Caso 2} & \multicolumn{3}{|c|}{ Caso 3} & \multicolumn{3}{|c|}{ Caso 4} \\
\hline & Início & Final & $\Delta$ & Início & Final & $\Delta$ & Início & Final & $\Delta$ & Início & Final & $\Delta$ \\
\hline MC (kg) & 43,5 & 45,1 & 1,6 & 26,8 & 26,4 & $-0,4$ & 43,0 & 45,6 & 2,6 & 30,7 & 31,5 & 0,8 \\
\hline IMC (kg/m²) & 19,8 & 20,5 & 0,7 & 13,4 & 13,2 & $-0,2$ & 16,7 & 17,5 & 0,8 & 15,2 & 15,6 & 0,4 \\
\hline Percentil & 50 & 50 & - & 1 & 1 & - & 25 & 50 & - & 5 & 15 & - \\
\hline CFCS (pontos) & 33 & 21 & -12 & 26 & 20 & -6 & 41 & 20 & -21 & 32 & 21 & -11 \\
\hline CFFS (pontos) & 5 & 0 & -5 & 4 & 0 & -4 & 5 & 0 & -5 & 5 & 0 & -5 \\
\hline CVF \% & 32,6 & 35,0 & 2,4 & 46,9 & 65,0 & 18,1 & 50,4 & 66,9 & 16,5 & 55,8 & 52,3 & $-3,5$ \\
\hline $\operatorname{VEF}_{1 \%}$ & 16,7 & 24,0 & 7,3 & 26,1 & 55,0 & 28,9 & 36,3 & 52,5 & 16,2 & 28,7 & 28,1 & $-0,6$ \\
\hline PFE \% & 12,1 & 32,0 & 19,9 & 36,8 & 57,0 & 20,2 & 33,9 & 60,9 & 17 & 27,7 & 23,3 & $-4,4$ \\
\hline $\mathrm{FEF}_{25-75 \%}$ & 5,1 & 10,0 & 4,9 & 5,1 & 20,0 & 14,9 & 14,2 & 27,8 & 13,6 & 9,1 & 8,0 & $-1,1$ \\
\hline Z5\% & 250,0 & 229,5 & $-20,5$ & 217,9 & 161,5 & $-56,4$ & 360,0 & 225,0 & -135 & 189,6 & 183,3 & $-6,3$ \\
\hline R5\% & 166,1 & 166,1 & 0 & 121,1 & 94,7 & $-26,4$ & 157,1 & 100,0 & $-57,1$ & 144,3 & 134,4 & $-9,9$ \\
\hline R20\% & 109,3 & 120,9 & 11,6 & 80,8 & 80,8 & 0 & 97,7 & 79,1 & $-18,6$ & 100,0 & 97,9 & $-2,1$ \\
\hline X5\% & 428,6 & 214,4 & $-214,2$ & 384,6 & 253,8 & $-130,8$ & 414,3 & 228,6 & $-185,7$ & 162,5 & 228,6 & 66,1 \\
\hline
\end{tabular}

Legenda: São apresentados os dados individuais de cada caso. Início: início da internação; Final: final da internação; $\Delta$ : variação dos dados (final menos inicial); MC: massa corporal; IMC: índice de massa corporal; Percentil: percentil do IMC; CFCS: Cystic fibrosis clinical score; CFFS: Cystic fibrosis foundation score; \%: porcentagem do predito; CVF: capacidade vital forçada; VEF expiratório; $\mathrm{FEF}_{25-75}$ : fluxo expiratório forçado de 25 a 75\% da CVF; Z5: impedância a 5Hz; R5: resistência total das vias aéreas; R20: resistência central das vias aéreas; $\mathrm{X} 5$ : impedância a $5 \mathrm{~Hz}$.

Tabela 3. Características de repouso e respostas fisiológicas ao TLS no início e final da internação por EPA.

\begin{tabular}{|c|c|c|c|c|c|c|c|c|c|c|c|c|}
\hline \multirow{2}{*}{ Parâmetros } & \multicolumn{3}{|c|}{ Caso 1} & \multicolumn{3}{|c|}{ Caso 2} & \multicolumn{3}{|c|}{ Caso 3} & \multicolumn{3}{|c|}{ Caso 4} \\
\hline & Início & Final & $\Delta$ & Início & Final & $\Delta$ & Início & Final & $\Delta$ & Início & Final & $\Delta$ \\
\hline$F R i$ & 35 & 36 & 1 & 41 & 32 & -9 & 43 & 29 & -14 & 30 & 37 & 7 \\
\hline$F R f$ & 35 & 32 & -3 & 50 & 40 & -10 & $27^{*}$ & 32 & 5 & 47 & 45 & -2 \\
\hline $\mathrm{SpO} 2 \mathrm{i}$ & 96 & 93 & -3 & 95 & 95 & 0 & 95 & 96 & 1 & 95 & 96 & 1 \\
\hline SpO2f & 90 & 91 & 1 & 90 & 90 & 0 & 97 & 97 & 0 & 95 & 90 & -5 \\
\hline FC $\mathbf{i}$ & 109 & 109 & 0 & 115 & 115 & 0 & 106 & 96 & -10 & 112 & 113 & 1 \\
\hline FC f & 122 & 124 & 2 & 130 & 127 & -3 & 127 & 118 & -9 & 120 & 109 & -11 \\
\hline \%FC máx & 61,5 & 62,5 & 1 & 65,3 & 63,8 & $-1,5$ & 63,8 & 59,3 & $-4,5$ & 60,1 & 54,6 & $-5,5$ \\
\hline BORG i & 0 & 0 & 0 & 0 & 0 & 0 & 0 & 0 & 0 & 0,5 & 1 & 0,5 \\
\hline BORG $\mathbf{f}$ & 0 & 0 & 0 & 2 & 1 & -1 & 0 & 0 & 0 & 4 & 2 & -2 \\
\hline EPEC $\mathbf{i}$ & 0 & 0 & 0 & 0 & 0 & 0 & 1 & 1 & 0 & 1 & 1 & 0 \\
\hline EPEC $f$ & 1 & 1 & 0 & 2 & 1 & -1 & 4 & 1 & -3 & 2 & 3 & 1 \\
\hline Repetições & 15 & 25 & 10 & 40 & 50 & 10 & 15 & 22 & 7 & 11 & 17 & 6 \\
\hline
\end{tabular}

Legenda: São apresentados os dados individuais de cada caso. Início: início da internação; Final: final da internação; $\boldsymbol{\Delta}$ : variação dos dados (final menos inicial); i: dados iniciais antes do TLS; f: dados ao final do TLS; fr: frequência respiratória: SpO $_{2}$; saturação de pulso de oxigênio; fc: frequência cardíaca; \%fc máx: porcentagem da fc máxima predita para idade; BORG: escala modificada de BORG; EPEC: escala de percepção de esforço para crianças. *Adolescente apresentou tosse durante a contagem da fr.

início e final da internação. Houve melhora dos escores de exacerbação, bem como dos parâmetros do sistema respiratório ao final da internação.

O desempenho médio no TLS apresentou incremento de $20,2 \pm 13,3$ repetições para $28,5 \pm 14,7$ ao final da internação, e os parâmetros cardiorrespiratórios não apresentaram tantas alterações (tabela 3). Especificamente o caso 4, não apresentou aumento nos valores de espirometria e IOS ao fim do tratamento. Além disso, realizou menos repetições no TLS, tanto no início como no final da internação, em 
comparação aos outros casos, além de ter referido uma maior percepção de esforço.

\section{DISCUSSÃO}

Esse relato de série de casos evidenciou melhora dos parâmetros do sistema respiratório, avaliados pela espirometria e IOS, bem como da capacidade de exercício no TLS, após o período de internação para tratamento da EPA, que consiste em antibioticoterapia, suporte nutricional, mucolíticos e fisioterapia respiratória. A literatura aponta esse pacote terapêutico como responsável pela melhora do quadro pulmonar ${ }^{1,2}$, no entanto, até o momento, a avaliação da capacidade de exercício em adolescentes com FC em períodos de EPA, ainda é pouco explorada pelos pesquisadores.

A melhora da função pulmonar, especialmente o parâmetro de volume expiratório forçado no primeiro segundo $\left(\mathrm{VEF}_{1}\right)$, devido ao tratamento da EPA, está bem estabelecido na literatura, e já foram relatadas mudanças positivas também nos parâmetros oscilométricos ${ }^{1}$. Porém, a avaliação da capacidade de exercício na população pediátrica com diagnóstico de FC, em períodos de EPA, ainda é pouco explorada pelos pesquisadores.

Um estudo avaliou a capacidade de exercício de 28 crianças e adolescentes com FC em EPA, por meio do teste de campo Modified Shuttle Test (MST) ${ }^{8}$. A média de VEF ${ }_{1}$ no início da internação foi de $63 \%$ e observou-se melhora significativa desse e dos parâmetros de CVF e $\mathrm{FEF}_{25-75}$, além do desempenho no MST ao final da internação ${ }^{8}$. Entretanto, a aplicação do MST apresenta empecilhos no ambiente hospitalar. Trata-se de um teste de campo que requer um espaço de 10 metros para realização e implica em deslocamento de equipamentos, como de medicação e oxigênio. Além disso, indivíduos com FC podem apresentar bactérias multirresistentes e estarem em isolamento, o que impede a aplicação deste teste. Por isso, entender as repercussões do TLS durante a EPA é de grande importância, visto que é um teste de fácil aplicação, com menor custo e viável em qualquer espaço.

Alguns estudos utilizaram o TLS em adultos com FC em estabilidade clínica ${ }^{3,9}$. Um grupo da Bélgica utilizou esse teste para avaliar as propriedades de medida e a mínima diferença importante em 14 adultos com FC de gravidade moderada a severa, antes e após três semanas de reabilitação pulmonar ${ }^{3}$. Os autores identificaram incremento de cinco repetições como mínima diferença importante ${ }^{3}$. O teste também foi considerado válido e confiável para mensurar a capacidade funcional na doença, visto que apresentou forte correlação com o pico de $\mathrm{VO}_{2}$ no TECP ${ }^{3}$.

Esse mesmo grupo aplicou o TSL em adultos com FC para correlacionar com TCP, TC6 e força de quadríceps ${ }^{9}$. Houve correlação entre os parâmetros cardiorrespiratórios do TSL e do TCP, além disso, o número de repetições como produto da massa corporal (repetições x massa corporal) apresentou correlação positiva com força de quadríceps e o pico de $\mathrm{VO}_{2}$ do TCP $^{9}$. Porém, esses estudos não avaliaram nenhuma intervenção, como por exemplo o tratamento da EPA.

Outro achado nos dados desta série de casos, foi a manutenção dos parâmetros cardiorrespiratórios no TLS entre o início e o final da internação, sem grandes mudanças na SpO2 e percepção de esforço. Cox et al. também não encontraram mudanças significativas na fc, SpO2 e percepção de esforço avaliada pelo EPEC, o que não foi discutido pelos autores ${ }^{8}$. Esse evento parece indicar que os adolescentes tiveram um maior número de repetições no TLS -melhor desempenho- às custas da mesma demanda cardiorrespiratória, sendo que nenhum dos casos avaliados atingiu pelo menos $90 \%$ da fc máxima, ou seja, a capacidade máxima de exercício, tanto no início como no final da internação ${ }^{10}$. Porém, houve redução na porcentagem da fc máxima no fim da internação, o que sugere melhora da capacidade de exercício.

Investigações com maior tamanho amostral são necessárias, visto que uma das limitações do presente estudo foi o pequeno número de casos coletados, o que inviabilizou a condução de uma análise estatística mais apurada. Aplicou-se também apenas um TSL, devido ao estado clínico de EPA dos participantes. Ainda, futuras pesquisas podem incluir a análise de gases para avaliação das repercussões fisiológicas do TLS durante a EPA.

\section{CONCLUSÃO}

Nesta série de relatos de casos, evidenciou-se que o tratamento da EPA em adolescentes com FC desencadeou uma resposta positiva no quadro clínico desses indivíduos, com melhora de parâmetros de função pulmonar e mecânica respiratória, e da capacidade de exercício avaliada pelo TLS.

\section{FONTES DE FINANCIAMENTO}

Fundação de Amparo à Pesquisa e Inovação do Estado de Santa Catarina, FAPESC/Brazil (PAP UDESC, Chamada Pública Nº4/2018, Termo de Outorga 2019TR658).

Coordenação de Aperfeiçoamento de Pessoal de Nível Superior (CAPES).

\section{CONFLITO DE INTERESSE}

Nada a declarar.

\section{REFERÊNCIAS}

1. ASSOBRAFIR: Associação Brasileira de Fisioterapia Cardiorespiratória e Fisioterapia em Terapia Intensiva. Recomendação Brasileira de Fisioterapia na Fibrose Cística: Um guia das boas práticas clínicas. Assobrafir Ciência. 2019

2. Cystic Fibrosis Trust. Standards of Care and Good Clinical Practice for the Physiotherapy Management of Cystic Fibrosis. London: Cyst Fibros Our Focus. 2020 
3. Radtke T, Puhan MA, Hebestreit $\mathrm{H}$, Kriemler S. The 1-min sit-to-stand test-A simple functional capacity test in cystic fibrosis? J Cyst Fibros. 2016;15(2):223-6. http://dx.doi. org/10.1016/j.jcf.2015.08.006. PMid:26363563.

4. De Assumpção MS, Gonçalves RM, Martins R, Bobbio TG, Schivinski CIS. Reference equations for impulse oscillometry system parameters in healthy Brazilian children and adolescents. Respir Care. 2016;61(8):1090-9. http://dx.doi. org/10.4187/respcare.04226. PMid:27165421.

5. Polgar G, Promadhat V. Pulmonary function testing in children: techniques and standards. Philadelphia: Saunders; 1971. 273 p.

6. Knudson RJ, Slatin RC, Lebowitz MDBB, Burrows B. The maximal expiratory flow-volume curves. Normal standards variability and effect of age. Am Rev Respir Dis. 1976;113(5):587-600. PMid:1267262.
7. Machado FA, Denadai BS. Artigo original validade das equações preditivas da frequência cardíaca máxima. Arq Bras Cardiol. 2011 Ago;97(2):136-40. http://dx.doi. org/10.1590/S0066-782X2011005000078. PMid:21739069.

8. Cox NS, Follett J, McKay KO. Modified shuttle test performance in hospitalized children and adolescents with cystic fibrosis. J Cyst Fibros. 2006;5(3):165-70. http://dx.doi. org/10.1016/j.jcf.2006.02.004. PMid:16679070.

9. Radtke T, Hebestreit $H$, Puhan MA, Kriemler S. The 1-min sitto-stand test in cystic fibrosis: insights into cardiorespiratory responses. J Cyst Fibros. 2017;16(6):744-51. http://dx.doi. org/10.1016/j.jcf.2017.01.012. PMid:28188010.

10. Karila C, de Blic J, Waernessyckle S, Benoist M-R, Scheinmann P. Cardiopulmonary exercise testing in children an individualized protocol for workload increase. Chest. 2001;120(1):81-7. http://dx.doi.org/10.1378/chest.120.1.81. PMid:11451820. 